\title{
Personalized Gaming for Motivating Social and Behavioral Science Participation
}

\author{
Casper Harteveld \\ Northeastern University \\ Boston, MA, USA \\ c.harteveld@northeastern.edu
}

\author{
Steven C. Sutherland \\ University of Houston-Clear Lake \\ Houston, TX, USA \\ sutherland@uhcl.edu
}

\begin{abstract}
Game-like environments are increasingly used for conducting research due to the affordances that such environments offer. However, the problem remains that such environments treat their users equally. In order to address this, personalization is necessary. In this paper we discuss the need to personalize gamified research environments to motivate participation by illustrating a playful platform called Mad Science, which is being developed to allow users to create social and behavioral studies. This discussion is both informed by the platform's affordances and use thus far as well as existing theories on player motivation, and contributes to theory-informed approaches to (gamified) personalization technologies.
\end{abstract}

\section{ACM Classification Keywords}

H.5.m. Information Interfaces and Presentation (e.g. HCI): Miscellaneous; I.6.8. Simulation and Modeling: Types of Simulation-Gaming; K.4.m. Computers and Society: Miscellaneous

\section{Author Keywords}

experimental research; gamification; motivation; personalization.

\section{INTRODUCTION}

Our world is becoming increasingly reliant on technology to achieve the goals that were previously only possible in physical spaces and with humans guiding how those goals would be achieved. For teaching, this has meant that students have often been passengers in their own educational experiences with teachers determining what would be learned and how that information would be made accessible [10]. This has often meant that all students in a classroom receive the same instruction and lessons regardless of how best they learn, what they already know, and what they are capable of learning. This one-size-fits-all process is similar in research. Participants show up to a study, not knowing what the study is about. The researcher determines what the study is about, how and where it will be administered, and what the benefits and incentives,

Permission to make digital or hard copies of all or part of this work for personal or classroom use is granted without fee provided that copies are not made or distributed for profit or commercial advantage and that copies bear this notice and the full citation on the first page. Copyrights for components of this work owned by others than ACM must be honored. Abstracting with credit is permitted. To copy otherwise, or republish, to post on servers or to redistribute to lists, requires prior specific permission and/or a fee. Request permissions from permissions@acm.org.

HUMANIZE'17, March 13 2017, Limassol, Cyprus

(C) 2017 ACM. ISBN 978-1-4503-4905-5/17/03 . .\$15.00

DOI: http://dx.doi.org/10.1145/3039677.3039681 if any, are available for participation. Every participant participates in the same location and are often drawn from very similar backgrounds (homogeneity of samples) [24].

The problem is that the way research has been conducted in the past is such that the participants that are meant to be involved are all treated equally, regardless of individual differences, and as passengers in someone else's plan [24]. However, we know that individuals respond very differently to incentives and different motivators might be more or less motivating for different participants $[17,8,16]$. One participant may try their best just to give it everything they have, others participate for course credit and only show up to ensure they receive it, others respond to financial incentives, while still others may respond to scores or praise. Some participants may feel anxious in a physical lab and their results might not reflect their ability. We therefore need to rethink our approach, ensuring research considers the participants in the design of studies and how to ensure participants of different backgrounds are included in their samples. It is difficult for humans to consider and modify their work for every participant; however, with advances in technology and the use of virtual experiences, these challenges become easier to address. When designed appropriately, technology can quickly identify user characteristics and modify complex content or provide assistance to increase the likelihood the intended goals of the experiences are achieved.

We seek to develop a personalized technology that empowers users to participate and create social and behavioral experiments, leveraged by gaming technologies and techniques, and thereby transforms research methods education and advances the social and behavioral sciences, specifically in the use of experimental research. To develop this technology, we have been creating a playful platform called Mad Science and have evaluated its potential by replicating experiments $[22,37]$ and by letting students create experiments using a paper prototype [23]. We then built a digital prototype iteratively based on these initial insights and prior game development experiences [21] and have recently used these digital prototypes in a number of classrooms [20].

In this paper we discuss the need to personalize a platform such as Mad Science by using this platform as a case study. The main research question underlying this effort concerns "What are the possible design considerations for personalizing a playful platform for experimental research such as Mad Science to motivate participation (both playing as well creating experiments)?" The resulting discussion presented in 
this paper is both informed by the platform's affordances and use thus far as well as existing theories on player motivation, and contributes to theory-informed approaches to (gamified) personalization technologies.

\section{BACKGROUND}

Before we detail the Mad Science platform, we will describe here why we believe that game-like environments such as Mad Science are promising for experimental research, and what role personalization may play. Additionally, we describe what work inspired and led to Mad Science.

\section{Affordances of Game-Like Research Environments}

The idea of the Mad Science platform is based on the emerging use of games and game-like environments as a research method [1,7]. Clear affordances that such environments offer for research are to: (a) immerse people in authentic situations, including situations that are difficult to observe in reality; (b) retrieve rich behavioral data in an unobtrusive but controlled manner; and (c) engage a broad global audience over a sustained period of time. The first affordance, immersing participants, is of importance because participants may not respond authentically when they are put in artificial environments and are engaged with paper-and-pencil tasks that do not have any real consequences. A game, albeit virtual, is more ecologically valid because players have goals they need to achieve and their actions do have real consequences [40]: they either win or lose. In addition, players are situated in an actual context rather than the traditional laboratory experiments that have nothing to do with their task.

Regarding the second affordance, a rich array of behaviors are produced in games, which may otherwise require costly and invasive wearable sensors and cameras to track the same behaviors in natural environments. As games can be customized, researchers can both exactly determine what players experience as well as easily change aspects-assuming they have access to the software and the skills to make these changes. In a way, games provide the richness of the natural environment combined with the control of laboratory experiments.

As for the third affordance, participants may be more incentivized to engage with tasks and do not need to receive monetary incentives [29]. Such engagement is not only of importance to measure more natural responses but also to engage participants with tasks that require significant time. Because games can be played anytime and anywhere, it broadens the sample population, and the ability to do it across the world opens up all kinds of new scientific opportunities. In addition, games are increasingly using personalization strategies because this is considered key to improving player experiences [2]. This means that with game-like environments participants may not only be more (naturally) incentivized, there is also the possibility to move away from the one-size-fits-all approach akin to many social and behavioral studies, which assumes that each participant is equally motivated.

Of course, it should be noted that personalizing the experience of experiments has possible unforeseen side-effects, and may, in fact, confound the results. By actually considering personalization as an independent variable this potential bias can be addressed. Researchers should also be thoughtful about what is being personalized. For example, with Mad Science it is possible to personalize the platform (i.e., points for participating in an experiment or access to a secret area) or the experiments (i.e., get recognition or being able to open a door).

Despite the clear promise of game-like environments for research, this use is still very sparse and more research is warranted. The sparse use might be because of perceived issues of validity, in spite of the ecological validity argument and that research thus far shows that, for the most part, individuals behave similarly in both virtual and real environments [6]. Also, it should be considered that today's interactions happen mostly digitally. The sparse use might also be attributed to the lack of accessibility to easily create game-based experiments, an issue Mad Science attempts to address. Finally, what may increase the interest to invest in game-like technologies is when it is clearly demonstrated that personalization of research matters. If personalization matters, there is a clearer need to make use of technologies that can adapt to individual users and that can scale to include thousands of participants to account for the variability created by the personalization technologies.

\section{Inspirations for Game-Like Research Environments}

Various examples of playful environments exist that have been developed successfully to support scientific inquiry for various disciplines, in particular for STEM topics and much less so for social and behavioral science, such as Quest Atlantis [3] and WhyVille [26]. Some are also "learner-driven" and with that we mean that the experience is not pre-defined by the designers but that the learners themselves define what they want to engage in and how they go about doing this-with the support of the environment. However, even these learner-driven environments do not provide authoring tools to conduct research by its users, certainly not for virtual research experiments.

Regarding the creation of experiments, various software exists in psychology, which allow users to implement experiments, such as E-Prime [35] and PsyToolkit [36]. Despite that some have a graphical user interface, such software is not attuned to non-programmers and specifically supports the creation of tasks that lack any kind of context. In addition, they are intended to be deployed in physical lab settings, though arguments are made to put these experiments online and environments are created where this can be done in a crowdsourced manner, such as Volunteer Science [27]. The goal for Mad Science is to be both a research and educational tool and enable the next generation of virtual experiments, which are easily created, contextualized, crowdsourced, collaborative (through "remixing" experiments), and gamified.

In terms of authoring tools, related work has been done for programming and game design tools for non-technical audiences and Interactive Fiction (IF). Efforts such as Scratch [33] or Alice [13] use a general-purpose language, simplified into a more friendly, graphical language to teach children how to program. Some game design tools, on the other hand, have drag-and-drop interfaces (e.g., Gamestar Mechanic [38] and Game Maker [31]). The idea of "scripting" a scenario bears much similarity with IF authoring tools, which typically allow for text-only interactive experiences, and also range in 
format, from dedicated programming languages (e.g., ChoiceScript [30]) to drag-and-drop interfaces (e.g., Twine [28]). We take inspiration from all of this work in developing a more dedicated, user-friendly environment for creating experiments.

It should be noted that researchers can harness all of the aforementioned authoring tools as well as games and game-like platforms that are accompanied with authoring tools or that provide capabilities to modify (called "modding"). For example, researchers have created a modification (or "mod") using an existing game to study personality in games [9]. In addition, as a proof-of-concept for Mad Science we initially used Twine in our classrooms to create experiments with. However, all of these environments are not necessarily dedicated to experiment creation nor do they provide the infrastructure to facilitate this process.

\section{THE MAD SCIENCE PLATFORM}

The Mad Science platform consists of building the following main components: the basic authoring tools, scenarios, personal lab, and a portal. It is intended to be used in higher education for teaching experimental research in a constructionist, experiential manner and by professional researchers for conducting the next generation of experimental research. To emphasize its playful nature, the platform is set within a lighthearted take on the mad scientist theme, where we conceive of a "mad scientist" as someone who is intrinsically curious and makes use of inventive and playful ways to explore human behavior. In this world of Mad Science, users join the corporation Mad Science Inc. as one of their new mad scientists and are provided with tools that allow them to create almost any kind of behavioral or social experimental scenarios visualized in a 2D virtual environment (Fig. 1). Currently, experiments are largely modified through the use of dialog and actions of objects/characters and player behavior is measured through the dialog or action choices they select for how their character will respond. Therefore, scenarios work best when they are able to utilize these types of variables and this functionality was the vision for the tools that are provided to players to create their experiments.

To exemplify a number of basic tools [20], users can design the player-character and the non-player characters with the character creator. Some characters may already exist, others may need to be modified or even developed from scratch. Similarly, with the scene creator existing settings can be picked or modified, and entirely new settings will need to be made from scratch (Fig. 2). The scene creator acts much like a theater stage where users can place objects, including characters, into a setting by picking them from a list of objects. The third tool concerns the scriptor, which allows users to make a visual decision tree for how a scenario unfolds and to specify the dialog and actions (e.g., move a character, play a sound) as well as how performance is evaluated (through variables). Then, the fourth tool is the manipulator, where users can make scenario variations by changing text, characters, settings, or a combination thereof. The tool registers the differences between the different conditions so it is clear what manipulations have been included. In addition to these basic authoring tools, users get empowered with tools to create briefings/debriefings (descriptor) and specify what data to collect (collector).

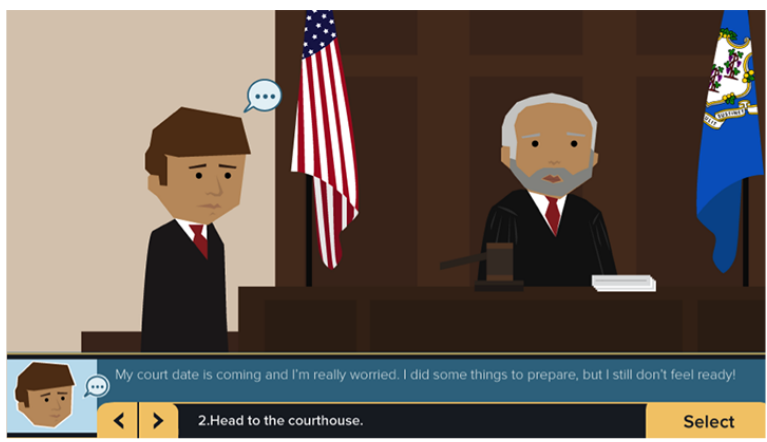

Figure 1. Example of an immersive experiment. Dialog choices are made at the bottom part of the interface. ONortheastern University

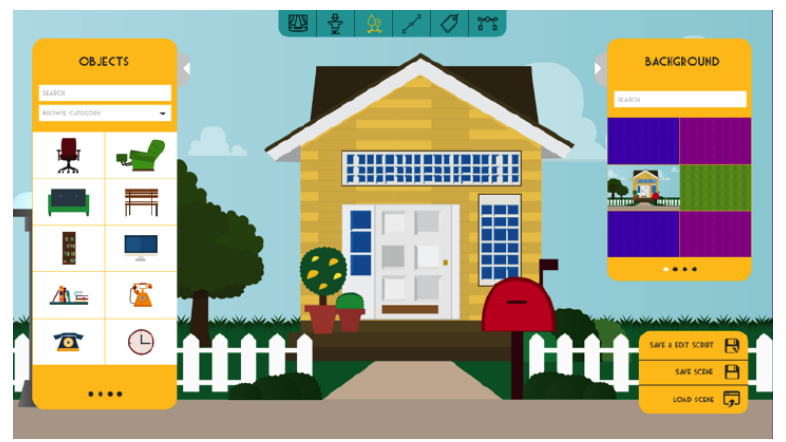

Figure 2. An example of an authoring tool. This is the scenario creator that enables users to create the settings for experiments. ONortheastern University

Once a scenario is finished, it will be embedded into a webpage on the portal, a website that hosts all of the scenarios. Each scenario webpage provides statistics about the scenario, including a rating and number of plays. Users can share and distribute the webpage over social media to invite people to play. Although experiments are only able to be created and made available by users with a registered Mad Science account, invited players can participate in the study (playing the scenario) as guests or as registered users on the portal. All data is logged and made available to both the creators as well as the players through the personal lab, which is a personal webpage for each registered user. Additionally, to foster collaboration, the scenarios themselves become available to players through the personal lab, making it easy to make modifications and run new studies. Players will be provided the ability to contact the study creators for studies they have completed. Opening this dialog between researchers/players will facilitate collaboration for players wanting to modify studies. The personal lab will have user stats, achievements, and socializing features-in essence all kinds of incentives to engage with the platform, and that can be personalized.

Thus far, we have developed the basic authoring tools and a basic portal website that hosts our experiments, including a number of classical decision making experiments that we replicated ourselves, such as the framing and decoy effect 
[37]. While recreating experiments, we thought of how they would translate to a game-based format [22]. For the framing effect [39], participants are immersed in a game show where they answer a number of questions to gain money and then decide how much they give to a friend. At the end of this "Gifts-to-Give game show", players decide what they do with their gained money, and their options are either framed negatively ("Your friend loses X of your money earned") or positively ("Your friend wins X of your money earned"). For the decoy effect, which is the phenomenon where consumer preferences are changed when presented with a third option that is asymmetrically dominated [25], we immersed players into a scenario where they have to purchase a boat. They get three options, which differ in cost and time to build. In one condition, the third option acts as a decoy, in another it does not. We were able to replicate the framing effect but failed to replicate the decoy effect because player choices weremuch to our surprise-dominantly affected by cost, despite the virtual nature of the currency, and that time did not impact decisions even though players were required to wait until their boat was finished (a real world consequence). However, this failure provided valuable insights into gamifying experiments and specifically revealed the promise of virtual money as an incentive.

We also implemented the platform in a number of courses in higher education. Our observations are that students are hesitant at first, especially if they do not have any prior game design or programming experience, and have problems with important facets of experiment creation, from hypothesis generation to random assignment $[23,20]$, which suggests a need for intelligent assistance. However, we also observed that students are engaged with the creation of the playful experiments. One example is a student-created experiment based on the mimesis effect [15], which is the effect that players make in-game choices aligned with the role of their character. The students created an experiment where players get randomly assigned to either a good or an evil person. Then players find a "magic wand" and can make a wish. They discover that every time they use the magic wand their wishes come true but also something terrible happens in the world. After the second use of the magic wand, and it should have become clear what negative impact the magic wand has, players receive the choice to stop or keep using the wand. The students discovered that when players play as a good person their decision to stop differs; however, when they play as an evil person all of them keep using the wand until the bitter end.

\section{DISCUSSION}

It is clear that personalization would benefit playful platforms such as Mad Science but also other efforts that aim to gamify practices, be it exercise or civic engagement. Although the need to personalize games to motivate players to initially begin playing and then to encourage players to return and continue playing is necessary for games in general to be successful [2], the issue of motivating players to play a non-entertainment game is of critical importance, especially if its success is reliant on a large number of participants. For example, with Mad Science the experiments that are designed will fail to effectively analyze differences between experimental conditions without a sufficient number of participants. As discussed, there may also be other reasons than scale and sustainability. In the case of Mad Science, personalization is also necessary to advance the social and behavioral sciences by being able to move away from a one-size-fits-all approach.

However, it is somewhat unclear how personalization can be pursued and in what manner-and the topic of motivation in the context of non-entertainment games is not well understood [11]. Most personalization efforts in games focus on player psychology [2], in particular motivation but also player types/personalities, and game difficulty, specifically built around the theory of flow [12]. Here we discuss the theories and approaches we are initially intending to draw from and how those can be translated to an implementation in Mad Science. Future efforts will need to ascertain what theories and related designs are most effective in engaging participants, and what analytical approaches are necessary to profile players.

\section{Theories of Motivation}

One of the affordances of game-like environments is to engage users. But similar to research, we cannot assume that users are motivated in the same way when they play. One of the early accounts for player differentiation are Bartle's player types [5]. Based on observations in Massively Multiplayer Online Games (MMORPGs), four player types were identified: achievers, explorers, socializers, and killers. Achievers thrive on prestige and accomplishing goals; explorers on discovering areas, role-playing, and creation; socializers on interacting with others (real or virtual); and killers on competition, in particular frustrating or beating other players.

Additional survey research confirmed three of the Bartle types by suggesting that players are primarily incentivized through achievement, immersion, or socializing [41]. The three confirmed player motivations/types are also closely associated with the three basic needs from the popular macro theory on motivation referred to as self-determination theory [34], which has also been proposed as the basis for a motivational model for game engagement [32]. It distinguishes between competency (need to master environment and outcome, see achievement or achiever), autonomy (need to act out of our own interests and values, see immersion or explorer), and relatedness (need to interact with other people, see socializing or socializer). A meta-analysis on player types of all possible classifications further confirmed that most types can be classified into these three categories [18]. The other three less common categories that player types are based on are: domination (i.e., essentially Bartle's "killer" type), gaming intensity and skill (e.g., casual vs. hardcore player), and in-game demographics (e.g., avatar class and game server).

In our opinion, the distinction of in-game demographics is not so much a player type but rather illustrative that artifactual affordances are situated [14]. Situations may arise within the game itself, be it a character that the player embodies or the culture on a particular server or clan/guild. It can also be external to the game. For Mad Science, it differs whether participation is part of a class requirement, voluntary, or for financial remuneration. The situation a player is in changes 
the meaning of how the game elements are perceived and thus whether players are motivated and find the experience rewarding. Additionally, it matters how players interpret the situation itself. Prior research has shown that students in a classroom perceived playing an educational game differently: as either a playful or educational activity [4]. Therefore, motivation in games seems a complex interplay between player type, artifactual affordances, the situation, and then the player perception of both the artifactual affordances as well as the situation.

The theory of triadic game design [19] can help to shed further light on the complexity of how games can be experienced, especially if such games have a serious purpose such as Mad Science. This theory posits that there are three paradigms to be considered, the world of reality, meaning, and play. Each paradigm has its own people, disciplines, aspects, and criteria in designing games. However, this theory can also be used to look at player experience. First, players are people who have particular personal characteristics and interests ("players as person"). For Mad Science it matters if a person is risk-averse and has an interest in finance for an experiment on financial risk taking. Second, players make sense of the world in different ways ("players as meaning makers"). Their sense-making depends on the level of education, interest to learn or contribute, prior knowledge, and-as elaborated upon in the previous paragraph - their perceptions of the activity. Third, players are players who enjoy particular types of game experiences and play games in a particular way ("player as player"). This means we have to view players from multiple perspectives, not just from the perspective of the world of Play, something the literature in game research tends to do.

Both the player perspectives, as illustrated through triadic game design, as well as the situational/perceptual complexity of game playing are of importance to evaluate the intrinsic versus extrinsic motivation for players to engage. In short, extrinsic motivation is when behavior is driven by external rewards (e.g., grades, money); intrinsic motivation is when behavior is driven by internal rewards (e.g., I want to understand this topic). This distinction is relevant to game-like environments because intrinsic motivation is more powerful and extrinsic motivation is known to undermine intrinsic motivation but also to lead to intrinsic motivation [34]. What may be intrinsically or extrinsically motivating will depend on the player (and their situation). Participating in the research process may, in and of itself, be intrinsically motivating for a small group. However, for players who do not find this goal intrinsically motivating, there is a need for additional or completely separate elements to keep them engaged.

While theoretically there is a good reason to personalize experiences, the question that follows is how to design and analyze for the diversity of players. In this paper we focus our further discussion on the design, in particular in the context of $\mathrm{Mad}$ Science, but acknowledge that methods and techniques for profiling players are key to a game's success, and that this will have its own set of challenges. For example, how to determine the situation of play as well as player perceptions regarding the situation and the game itself is specifically challenging.

\section{Design for Motivation}

For a game designed for research to engage its users, game elements must provide motivational affordances. We use the motivational affordance framework previously proposed by Zhang [42] as a lens through which personalization might be approached and discuss this in the context of Mad Science. Zhang proposed ten design principles to meet five motivational needs, the first four which are related to Bartle's player types and the fifth to the aesthetic pleasure of playing playful experiences. These design principles, when used independently, increase the likelihood that the technology will create motivational affordances and thus increase the likelhood users will engage with it. When these principles are combined, and adjusted accordingly, there are much greater chances that a broad range of users will engage with it and for longer periods.

Design Principles 1 and 2 (support autonomy and promote creation and representation of self-identity, respectively; see explorer) are suggested to meet the psychological motivational needs for autonomy and self. Mad Science will provide users with the ability to choose which experiments they complete and which topics they explore. Additionally, the game will allow players to express themselves as individuals. The player's character, laboratory, environment, and research agenda will be based on the player's own design or modification, reflecting their identity. We expect that players will be motivated to spend time modifying their experience on the platform. To encourage this, players will be given more freedom to adjust their surroundings and their character as they participate in additional experiments.

Design Principles 3 and 4 (design for optimal challenge and provide timely and positive feedback, respectively; see achiever) are suggested to meet the cognitive motivational needs for competence and achievement. Creating experiments and participating in research are not everyday experiences for most people. If every experiment that a player participates in is an easy, limited experience, players may not feel inclined to continue to play. Experiments must be challenging, based on current player understanding, and provide relevant feedback to players throughout their participation and immediately following. This process will allow players in Mad Science to gain more complex skills as they progress, while maintaining increasing levels of difficulty. Challenge will also be accomplished across the platform by providing increased access to the authoring tools (through leveling) as players gain experience. If the goal is to motivate players to explore research, understanding how others approached their own studies is helpful and the reason we require studies to have debriefing information to tell participants what the purpose of the study was and, by providing access to the results and the code for the experiment, how the study was designed and whether it achieved its goals. Gaining access to this information and learning through experience is expected to motivate players.

Design Principles 5 and 6 (facilitate human-human interaction and represent human social bond, respectively; see socializer) are suggested to meet the social and psychological motivational need for relatedness. In the context of games for research, there is a need for the player to feel as though 
they are a part of the research community, rather than just an individual participant. We hope to accomplish this by having larger research labs, including multiple players, that have similar interests in research and by keeping players informed about the experiments they participated in and who has modified the player's own past experiments, encouraging the player to reach out to the new researcher to see the results of the modified study. This will allow players to follow the experiments they created or that they participated in and discuss research or other topics with members of the game's community. We expect to accomplish this using in-game chat rooms and lab spaces or using online forums on the game's website. Tracking progress and results from experiments that users participated in will be accomplished through the personal labs. Players will be more likely to continue to play and participate if they feel like they are a part of a larger community.

Design Principles 7 and 8 (facilitate one's desire to influence others and facilitate one's desire to be influenced by others, respectively; see killer) are suggested to meet the social and psychological motivational needs for leadership and followership. In a platform used to create and participate in research, we expect that these design principles are integral to the design. Players influence others and are influenced by others first by participating in experiments meant to measure influenced effects of dependent/manipulated variables. Players are additionally influenced by and influence others through the sharing of research findings and experimental design. The nature of the experimental process is such that it builds upon past studies and permits itself to be built upon by future studies. The ability to track the influence of one's work in this type of game is expected to encourage users to remain engaged to track their impact on the game.

Finally, Design Principles 9 and 10 (induce intended emotions via initial exposure to the game and induce intended emotions via intensive interaction with the game, respectively) are suggested to meet the emotional motivational needs for affect and emotion. The platform must use artistic styles that create an emotional reaction from the player. For Mad Science, this has meant designers forego realism for art that creates greater emotional responses from players. Additionally, the platform should increase the likelihood that players become immersed through gameplay. This last point is one of the hardest for which to design. There needs to be fluency between all of the tools and areas of the game. Any failure to provide cohesiveness introduces an opportunity for players to become distracted. This means that going from personal spaces into experiments needs to maintain some level of cohesion. We hope to accomplish this by providing the necessary balance between challenge and artificial intelligence (AI)-supported assistance to limit players becoming complacent or frustrated.

Maintaining appropriate levels of motivation is where AI support for personalization is most needed. An intelligent system should be designed to identify whether any of these needs are failing to be met, whether these needs are important motivations for a given player, and how to modify the experience, by introducing, highlighting, or modifying particular elements to increase motivation. Although the aforementioned suggested design elements provide motivational affordances, these elements are not introduced in isolation, rather they are situated in various contexts. Therefore, it is necessary to consider the element, the situation, and the interaction between the two when determining whether motivational needs will be or are being met [14]. Therefore, a truly intelligent system would need to look beyond player characteristics and learn to modify elements based on situational factors, such as whether the player is currently in the role of experiment designer, research participant, space modifier, or socializer. Additionally the player may be a volunteer or they may have been required to play by an outside influence (e.g., as a course requirement). All of these aspects might influence expectations and whether particular motivational affordances are possible.

\section{CONCLUSION}

Game-like environments have enormous potential for advancing social and behavioral science. In this paper we discussed that such environments can (a) immerse people in authentic situations, (b) retrieve rich behavioral data in an unobtrusive but controlled manner, and (c) engage a broad global audience over a sustained period of time. However, we also discussed that such environments have a need for personalization in order to truly engage participants and not apply a one-size-fits-all approach. We elaborated on several theories of motivation that can help to personalize playful technologies, and came to the conclusion that motivation is a complex dynamic process that requires considering various player perspectives as well as both the situational context and the player's perception of the experience. Using Zhang's [42] framework for motivational affordances we structured our discussion on how our insights can be applied to the playful platform called Mad Science, which aims to advance social and behavioral science, specifically for experimental research. Future research will take place that evaluates motivational theories and affordances for their effectiveness of personalizing playful experiences.

\section{ACKNOWLEDGEMENTS}

We thank everyone who contributed to the design of the platform. We further acknowledge the grant support from Northeastern University, Legal Services Corporation (LSC-TIG \#14035), and DARPA (D16AP0011).

\section{REFERENCES}

1. William Sims Bainbridge. 2007. The scientific research potential of virtual worlds. Science 317, 5837 (2007), 472-476.

2. Sander Bakkes, Chek Tien Tan, and Yusuf Pisan. 2012. Personalised gaming: A motivation and overview of literature. In Proceedings of the 8th Australasian Conference on Interactive Entertainment: Playing the System. ACM.

3. Sasha Barab, Michael Thomas, Tyler Dodge, Robert Carteaux, and Hakan Tuzun. 2005. Making learning fun: Quest Atlantis, a game without guns. Educational Technology Research and Development 53, 1 (2005), 86-107.

4. Jacqueline Barnes and Casper Harteveld. 2016. When is a game not a game? Considering player perceptions of an 
educational game. In Games + Learning + Society. ETC Press.

5. Richard Bartle. 1996. Hearts, clubs, diamonds, spades: Players who suit MUDs. Journal of MUD research 1, 1 (1996), 19.

6. Jim Blascovich and Jeremy Bailenson. 2011. Infinite reality: Avatars, eternal life, new worlds, and the dawn of the virtual revolution: HarperCollins, New York, NY.

7. Eduardo Calvillo-Gámez, Jeremy Gow, and Paul Cairns. 2011. Introduction to special issue: Video games as research instruments. Entertainment Computing 2, 1 (2011), 1-2.

8. Colin F Camerer and Robin M Hogarth. 1999. The effects of financial incentives in experiments: A review and capital-labor-production framework. Journal of risk and uncertainty 19, 1-3 (1999), 7-42.

9. Alessandro Canossa, Jeremy B Badler, Magy Seif El-Nasr, Stefanie Tignor, and Randy C Colvin. 2015. In your face $(\mathrm{t})$ : Impact of personality and context on gameplay behavior. In Proceedings of the Foundations of Digital Games.

10. Allan Collins and Richard Halverson. 2009. Rethinking education in the age of technology: The digital revolution and schooling in America. Teachers College Press.

11. Kevin Crowston and Nathan R Prestopnik. 2013. Motivation and data quality in a citizen science game: A design science evaluation. In System Sciences (HICSS), 2013 46th Hawaii International Conference on. IEEE, $450-459$

12. Mihaly Csikszentmihalyi. Flow and the psychology of discovery and invention. HarperCollins, New York, NY.

13. Wanda P Dann, Stephen Cooper, and Randy Pausch. 2011. Learning to Program with Alice. Prentice Hall Press.

14. Sebastian Deterding. 2011. Situated motivational affordances of game elements: A conceptual model. In ACM CHI 2011 Workshop, Gamification: Using Game Design Elements in Non-Gaming Contexts.

15. Ignacio X Domínguez, Rogelio E Cardona-Rivera, James K Vance, and David L Roberts. 2016. The mimesis effect: The effect of roles on player choice in interactive narrative role-playing games. In Proceedings of the 2016 CHI Conference on Human Factors in Computing Systems. ACM, 3438-3449.

16. Marylène Gagné and Edward L Deci. 2005. Self-determination theory and work motivation. Journal of Organizational behavior 26, 4 (2005), 331-362.

17. Lindsay T Graham and Samuel D Gosling. 2013. Personality profiles associated with different motivations for playing World of Warcraft. Cyberpsychology, Behavior, and Social Networking 16, 3 (2013), 189-193.

18. Juho Hamari and Janne Tuunanen. 2014. Player types: A meta-synthesis. Transactions of the Digital Games Research Association 1, 2 (2014).
19. Casper Harteveld. 2011. Triadic game design: Balancing reality, meaning and play. Springer, London, UK.

20. Casper Harteveld, Nolan Manning, Farah Abu-Arja, Rick Menasce, Dean Thurston, Gillian Smith, and Steven C Sutherland. 2017. Design of playful authoring tools for social and behavioral science. In Proceedings of the International Conference on Intelligent User Interfaces. ACM.

21. Casper Harteveld, Steven C Sutherland, and Yetunde Folajimi. 2016. Sustainable life cycle game design: Mixing games and reality to transform education. In Proceedings of Joint Conference of Digital Games Research Association and Foundations of Digital Games.

22. Casper Harteveld, Steven C Sutherland, and Gillian Smith. 2015. Design considerations for creating game-based social experiments. In ACM CHI 2015 Workshop, Researching Gamification: Strategies, Opportunities, Challenges, Ethics. Seoul, South Korea.

23. Casper Harteveld, Steven C Sutherland, Gillian Smith, Cigdem Talgar, and Amy J. Stahl. 2016. Standing on the shoulders of citizens: Exploring gameful collaboration for creating social experiments. In 49th Hawaii International Conference on System Sciences (HICSS). IEEE Press, Kauai, Hawaii.

24. Joseph Henrich, Steven J Heine, and Ara Norenzayan. 2010. The weirdest people in the world? Behavioral and brain sciences 33, 2-3 (2010), 61-83.

25. Joel Huber, John W Payne, and Christopher Puto. 1982. Adding asymmetrically dominated alternatives: Violations of regularity and the similarity hypothesis. Journal of consumer research 9, 1 (1982), 90-98.

26. Yasmin B Kafai. 2010. World of Whyville: An introduction to tween virtual life. Games and Culture 5, 1 (2010), 3-22.

27. Brian Keegan, Katherine Ognyanova, Brooke Foucault Welles, Christoph Riedl, Ceyhun Karbeyaz, Waleed Meleis, David Lazer, Jason Radford, and Jefferson Hoye. 2014. Conducting Massively Open Online Social Experiments with Volunteer Science. In Second AAAI Conference on Human Computation and Crowdsourcing.

28. Chris Klimas. 2009. Twine: An open-source tool for telling interactive, nonlinear stories. (2009). http://twinery.org/

29. Andrew Mao, Ece Kamar, Yiling Chen, Eric Horvitz, Megan E Schwamb, Chris J Lintott, and Arfon M Smith. 2013. Volunteering versus work for pay: Incentives and tradeoffs in crowdsourcing. In First AAAI Conference on Human Computation and Crowdsourcing.

30. Choice of Games. 2010. Introduction to ChoiceScript. (2010). https://www . choiceofgames.com/ make-your-own-games/choicescript-intro/ 
31. Mark Overmars. 2004. Teaching computer science through game design. Computer 37, 4 (2004), 81-83.

32. Andrew K Przybylski, C Scott Rigby, and Richard M Ryan. 2010. A motivational model of video game engagement. Review of general psychology 14, 2 (2010), 154.

33. Mitchel Resnick, John Maloney, Andrés Monroy-Hernández, Natalie Rusk, Evelyn Eastmond, Karen Brennan, Amon Millner, Eric Rosenbaum, Jay Silver, Brian Silverman, and Yasmin Kafai. 2009. Scratch: Programming for all. Commun. ACM 52, 11 (2009), 60-67.

34. Richard M Ryan and Edward L Deci. 2000. Self-determination theory and the facilitation of intrinsic motivation, social development, and well-being. American psychologist 55, 1 (2000), 68.

35. Walter Schneider, Amy Eschman, and Anthony Zuccolotto. 2002. E-Prime: User's guide. Psychology Software Incorporated.

36. Gijsbert Stoet. 2010. PsyToolkit: A software package for programming psychological experiments using Linux. Behavior Research Methods 42, 4 (2010), 1096-1104.
37. Steven C Sutherland, Casper Harteveld, Gillian Smith, Joseph Schwartz, and Cigdem Talgar. 2015. Exploring digital games as a research and educational platform for replicating experiments. In NEDSI Conference. Boston, MA.

38. Katie Salen Tekinbas, Melissa Gresalfi, Kylie Peppler, Rafi Santo, and James Paul Gee. 2014. Gaming the system: Designing with Gamestar Mechanic. MIT Press, Cambridge, MA.

39. Amos Tversky and Daniel Kahneman. 1985. The framing of decisions and the psychology of choice. In Environmental Impact Assessment, Technology Assessment, and Risk Analysis. Springer, 107-129.

40. Noah Wardrip-Fruin, Michael Mateas, Steven Dow, and Serdar Sali. 2009. Agency reconsidered. Breaking New Ground: Innovation in Games, Play, Practice and Theory. Proceedings of DiGRA 2009 (2009).

41. Nick Yee. 2006. Motivations for play in online games. CyberPsychology \& behavior 9, 6 (2006), 772-775.

42. Ping Zhang. 2008. Motivational affordances: Reasons for ICT design and use. Commun. ACM 51, 11 (2008), $145-147$. 